\title{
Evaluation of Spectroscopic Modeling for Iron Ions and Study on Non-Equilibrium Ionization Phenomena for Solar and LHD Plasmas*)
}

\author{
Tetsuya WATANABE, Hirohisa HARA, Norimasa YAMAMOTO ${ }^{1)}$, Daiji KATO $^{2)}$, \\ Hiroyuki A. SAKAUE ${ }^{2)}$ and Izumi MURAKAMI ${ }^{2)}$ \\ National Astronomical Observatory, 2-21-1 Osawa, Mitaka, Tokyo 181-8588, Japan \\ ${ }^{1)}$ Chubu University, 1200 Matsumoto-cho, Kasugai, Aichi 487-8501 Japan \\ ${ }^{2)}$ National Institute for Fusion Science, 322-6 Oroshi-cho, Toki, Gifu 509-5292, Japan
}

(Received 19 November 2012 / Accepted 13 May 2013)

\begin{abstract}
Spectroscopic observations of EUV emission lines in the transition region (TR) and the corona provide unique information on physical conditions in the outer atmosphere of the Sun. The EUV Imaging Spectrometer (EIS) on board the Hinode satellite is capable of observing, for the first time in EUV, spectra and monochromatic images of plasmas in the solar TR and corona; these plasmas could possibly be in non-ionization-equilibrium conditions. EIS observes over two-wavelength bands of $170-210 \AA$ and $250-290 \AA$, with typical time-resolutions of 1 - 10 seconds. Iron line emissions emerging from these wavelengths reveal that dynamic plasma accelerations and heating take place in the solar atmosphere. On the other hand, the tracer-encapsulated-pellet (TESPEL) experiments provide spectral information of EUV emission lines from iron ions produced in the Large Helical Device (LHD). Relatively cool plasmas with electron temperatures similar to those of the solar corona can be generated by controlling the neutral beam injector (NBI) system. A time-dependent collisional radiative (CR) model for elemental iron is developed as a common tool to diagnose temperatures and densities of those plasmas in the Sun and in LHD; no systematic model yet exists for iron ions in the L- and M-shell ionization stages, which are very important for coronal plasma diagnostics. Adopting the best available theoretical calculations, as well as generating the experimental data, we improve the atomic parameters of highly charged iron ions, and these results are used to extract more accurate diagnostic information out of the EIS spectra.
\end{abstract}

(C) 2013 The Japan Society of Plasma Science and Nuclear Fusion Research

Keywords: EUV emission line, iron ion modeling, non-ionization equilibrium, solar corona, LHD TESPEL experiment

DOI: $10.1585 /$ pfr.8.2501105

\section{Introduction}

The corona is a very extended and tenuous layer in the Sun's outer atmospheres, but is actually found to be very hot, being a few million $\mathrm{K}$ and having about a half of extra-nuclear electrons of iron stripped away. When ionized, iron, one of the most abundant elements in the universe, radiates in many emission lines over a wide range of wavelengths.

Spectroscopic observation of EUV emission lines in particular provides unique information on physical conditions in the outer atmosphere of the Sun. The Sun can be viewed as "a laboratory in space," which sometimes shows very interesting phenomena on a gigantic scale, that fascinate both plasma and solar physicists.

We report in this paper on an analysis tool developed to handle plasmas in non-equilibrium states, and this "time-dependent CR (collisional radiative)" model is ap-

author'se-mail: watanabe@uvlab.mtk.nao.ac.jp

*) This article is based on the invited presentation at the 22nd International Toki Conference (ITC22). plied, as a common tool, to both solar and laboratory plasmas. This model uses improved atomic datasets, selected both by evaluating theoretical calculations and via measurements in the laboratory.

The Hinode satellite observes the Sun from Earth orbit [1], and is leading to a better understanding of the dynamic and violent nature of solar coronal plasmas through high-sensitivity EUV spectroscopic observations conducted with its EUV Imaging Spectrometer (EIS) [2] (Fig. 1). High temperature iron plasmas are created in Large Helical Device (LHD) by injecting iron TESPEL (tracer-encapsulated-pellet). Modeling of iron ions is tested and improved with both solar and laboratory measurements. This is a good example for "multiple" cross validation of experiment/observation and modeling.

\section{Dynamism of the Solar Atmosphere}

Solar plasmas in the chromosphere, the transition region (TR), and the corona are intrinsically in nonionization equilibrium. The relaxation time scales to 


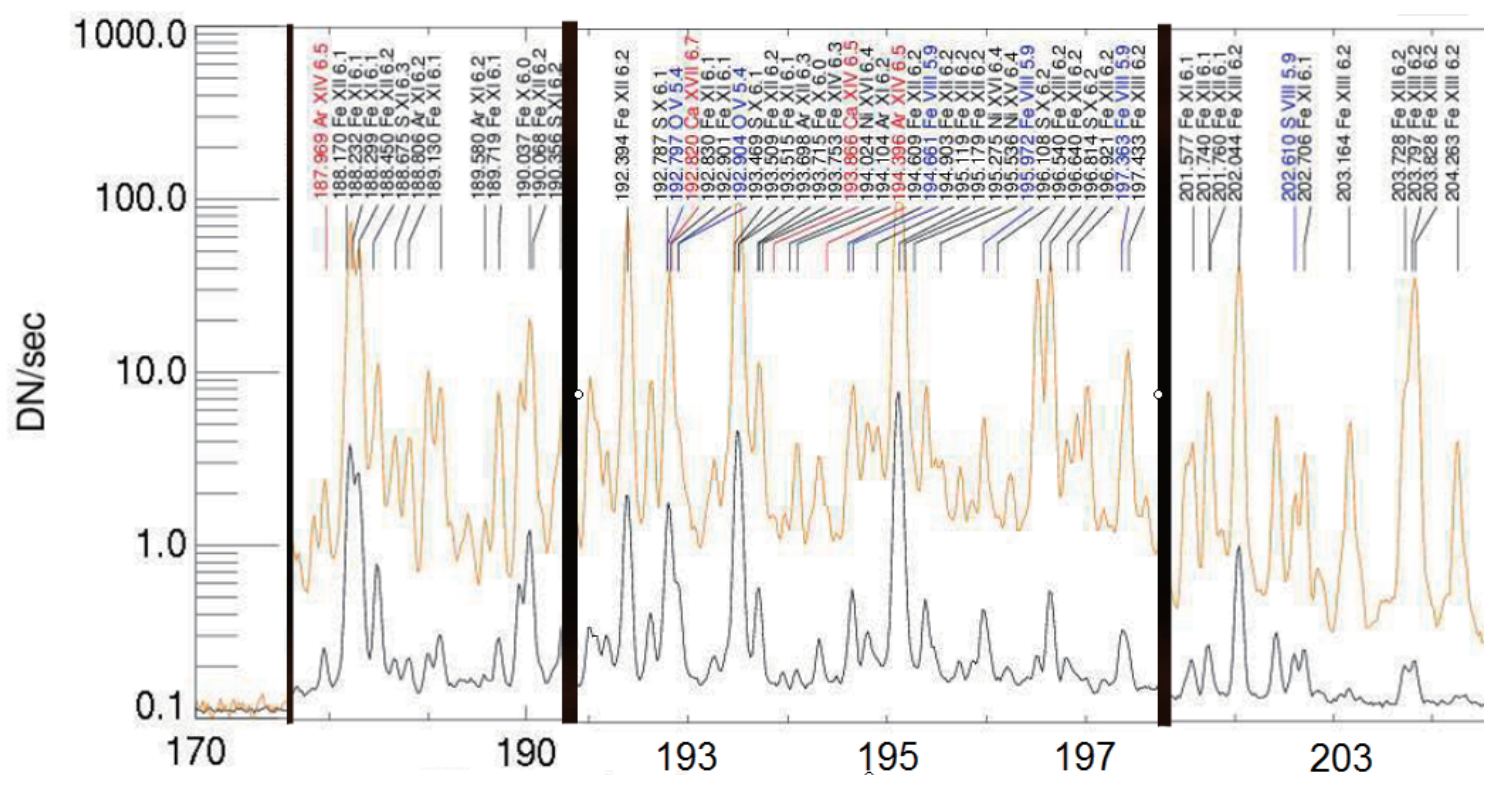

Fig. 1 EIS spectral atlas (partly enlarged) with line identification via CHIANTI database [3,4].

reach ionization equilibrium are simply estimated as $N_{\mathrm{e}} \times$ $\tau \sim 10^{12} \mathrm{~cm}^{-3}$ sec for the physical conditions in the solar outer atmosphere, where $N_{\mathrm{e}}$ is electron density and $\tau$ is relaxation time scale. If we substitute the observed density values for $N_{\mathrm{e}}$, the resulting time scales are $\tau \sim 10^{3} \mathrm{sec}$, for features of $10^{9} \mathrm{~cm}^{-3}$. With these timescales, transient ionization effects should have been detectable in solar data from not only EIS, but also from predecessors to EIS on spacecraft that flew years or even decades ago. In reality however, no such substantial non-equilibrium effects have been recognized. More specifically, previous analyses of solar spectra have almost always assumed ionization equilibrium, and those results have usually been more-orless consistent with each other, suggesting that there are no large deviations from non-thermal equilibrium in those observed spectra.

Why such expected deviations from equilibrium in the solar atmosphere have not been observed is unknown. It could be because the observed solar features are in a different physical state than we might expect. They might consist of so-far unresolved fine structures. These structures might be much denser than we can spatially resolve with our instruments. (Our instruments only observe much larger-scale, spatially-averaged structures, which overall have a lower density than the fine-scale structures. That is, the features our instruments to-date observe have a small "filling factor.") Those higher densities would mean the equilibrium timescale is quite small, small enough to explain that our observations and analyses to date show no large consequences of non-equilibrium conditions.

EIS might be able to observe the "tip of the iceberg" of these fine structures. Excess line broadenings of EUV emission lines originating from the TR and the corona have been known for a long time, but new EIS observations have taken important steps forward to understanding the mechanism of coronal heating and the triggering of solar flares (Fig. 2). From observational features subsonic upflow motions of tens of $\mathrm{km} \mathrm{sec}^{-1}$ and enhanced nonthermal velocities have been found near the footpoints of the AR loops, using a single Gaussian profile fits to EUV emission lines. When the same part of the active region is observed near the limb, both upflows and enhanced nonthermal velocities essentially reduce to zero. The outflows are positively correlated with nonthermal velocities in coronal plasmas $[5,6]$. Significant deviations from a single Gaussian profile are found in the blue wing of the line profiles for the upflows. These observations suggest that there are unresolved high-speed upflows in solar AR loop footpoints [5].

A significant coronal mass supply mechanism is found to be driven from the chromospheres below. The asymmetry of spectral lines observed with EIS identifies faint but ubiquitous upflows with velocities $\sim 50-100 \mathrm{~km} \mathrm{~s}^{-1}$ across a wide range of magnetic field configurations, and for temperatures from $10^{5} \mathrm{~K}$ to several MK. These upflows are spatially and temporally correlated with so-called "type II" spicules, which have similar upward velocities [7]. These upflows are pervasive and universal. The mass supplied by these spicule-like upflows can play a significant role in supplying the corona with hot plasma. This again suggests somewhat a different scenario for coronal heating, namely different from the "nanoflare" hypothesis, in which tiny and numerous flare occurrence in the corona is suggested to be responsible for coronal heating.

Concerning solar flares, EIS has found important diagnostic information around possible reconnection sites and loop-top high-temperature X-ray sources [8]: The 2007 May 19 flare was a small flare with its soft X-ray peak intensity barely reaching the GOES C-class level. However, 

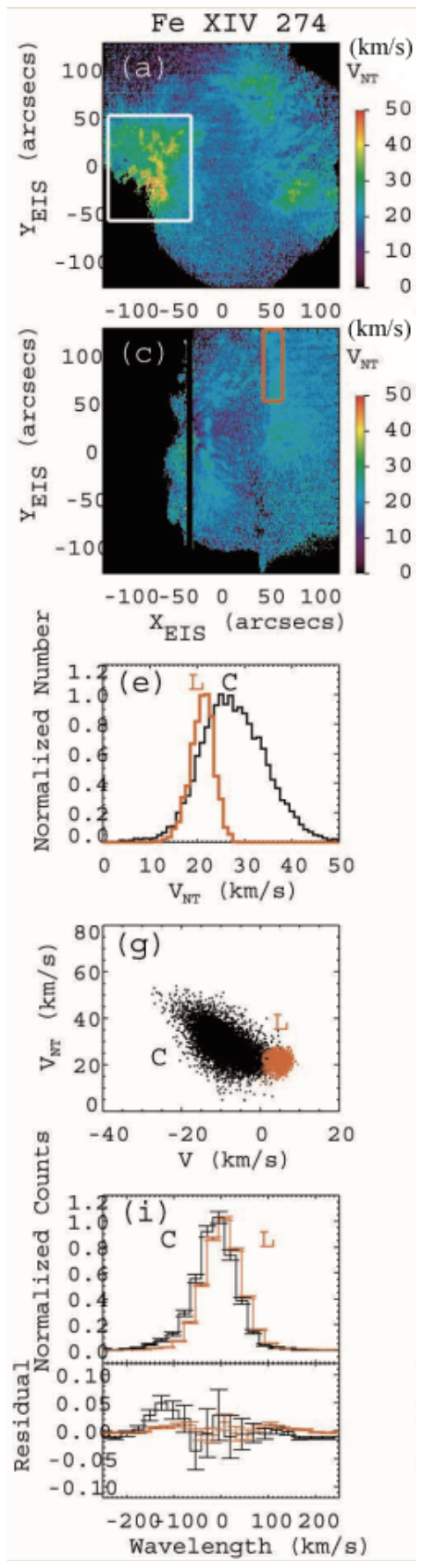

Fig. 2 Fe XIV $\lambda 274$ observations: Top to bottom: Nonthermalvelocity maps at disk center, (a); and at the west limb, (c); histograms of nonthermal velocity, (e); scatter plots between Doppler and nonthermal velocities, (g); and normalized line profile with their residuals from a single Gaussian fit at footpoint of a coronal loop, (i). C and $\mathrm{L}$ in (e), (g), and (i) indicate respectively observations at disk center and limb, within the white or red box in maps, of (a) and (c) [5].

the hot source observed in Fe XXIII and Fe XXIV emission lines has $T_{\mathrm{e}} \sim 12 \mathrm{MK}$ and $n_{\mathrm{e}} \sim 10^{10} \mathrm{~cm}^{-3}$. It shows excess line broadening, which exceeds the thermal Doppler width by $\sim 100 \mathrm{~km} \mathrm{~s}^{-1}$ with a weak redshift of $\sim 30 \mathrm{~km} \mathrm{~s}^{-1} \mathrm{~A}$ blueshifted faint jet is also observed and its line-of-site ve- locity exceeds $\sim 200 \mathrm{~km} \mathrm{~s}^{-1}$ with $T_{\mathrm{e}} \sim 9 \mathrm{MK}$. Coronal plasmas $\left(T_{\mathrm{e}} \sim 1.2 \mathrm{MK}, n_{\mathrm{e}} \sim 2.5 \times 10^{9} \mathrm{~cm}^{-3}\right)$ are pushed onto the loop-top region with a speed of $20 \mathrm{~km} \mathrm{~s}^{-1}$, and they disappear near the hot source, possibly heated to the temperature of the hotter faint jet. These observations can be explained only by magnetic reconnection that may take place near the loop-top region. Specifically, they also support the Petschek [9]-type magnetic reconnection with further evidence for the presence of the slow-mode and fast-mode MHD shocks in the reconnection [8].

\section{TESPEL Experiment in LHD with New EUV Spectrometer}

Laboratory experiments have been particularly useful for identifying specific problems in atomic models with independently calibrated density and temperature measurements. Highly charged iron ions are generated by injecting iron TESPELs (tracer-encapsulated-pellet) into the Large Helical Device (LHD) at the National Institute for Fusion Science (NIFS) in Toki.

Iron spectra are taken from these LHD experiments. Discharges in these shots last several seconds, and temperatures reach $2-3 \mathrm{keV}(\sim 20-30 \mathrm{MK})$ during the main part of discharge. Temperatures are then intentionally reduced to "coronal" temperatures via the injection of plastic pellets with iron cores (TESPELs), and during the plasma decay phases after the end of heating by neutral beam injection. The result is that iron line emissions at various ionization stages, from Fe VIII to Fe XXII, start to emerge more prominently, while emission lines from Fe XXIV dominate in the spectral region near $200 \AA$ during the main discharge before the injection.

These spectra are recorded via a flat-field EUV spectrometer with a varied-line-spacing groove grating (1200 grooves $\mathrm{mm}^{-1}$ at grating center) [10] developed to study emission spectra from medium-Z impurities in LHD. The spectrometer covers a wavelength range of 50 - $500 \AA$ using a laminar-type holographic grating, superior in suppressing the higher-order light, and in uniformity of sensitivity in the full wavelength range.

Spectral resolution of $\sim 0.24 \AA$ at $200 \AA$ is successfully achieved on the CCD detector $(0.198 \AA$ /channel $)$. A stack of aluminum foil is placed in front of the grating in order to screen out the emissions emerging at wavelengths shorter than $170 \AA$. This is necessary to avoid spectral contamination from higher energy iron lines that could show up in the second, third, or fourth order reflection. A new technique for the absolute calibration is implemented by combining the continuum radiation and a branching ratio of CIV lines.

\section{Modeling of Iron Ions}

\subsection{Collisional Radiative (CR) model}

New calculations based on the Hebrew UniversityLawrence Livermore Atomic Code (HULLAC) [11] are 
conducted. This code includes using the distorted-wave approximation to compute excitation cross sections, and the calculations are based on atomic data generated by the Dirac Atomic R-matrix Code (DARC) [12]. The population densities of the excited states and the spectral line intensities are calculated for Fe VII-XXVI ions. Explicit time-dependent calculations can be applicable by assuming a quasi-steady state solution, where the timedependence of the rate equations is explicitly taken into account only for ground levels and metastable levels, while that for excited states is not.

The new model we have constructed [13, hereafter Y08] is basically our original collisional radiative (CR) model for iron ions including the fine-structure levels up to $n=5$. This model includes processes of excitation and de-excitation by electron impact, radiative decay, radiative recombination, ionization, three-body recombination, and autoionization, as well as dielectronic capture and dielectronic recombination. Transition probabilities and cross sections of these processes were calculated with the HULLAC code [11]. The effective collision strengths calculated with R-matrix code and the radiative transition probabilities calculated by the GRASP code (GRASP6) [14] are used for the same transitions. The same Y08 model configuration replaced with radiative transition probabilities generated by the new code, GRASP13 [15], is also calculated to see the effects of data replacement [16]. The excitation rate coefficients for proton impact evaluated by Skobelev et al. [17] are also included for the transitions between fine structure levels of the ground-state configuration $[13,16,18]$.

Our measurements and observations are also compared to the results from the CHIANTI spectral modeling code [4].

\subsection{Case study - Fe XIII for density diagnos- tics -}

The silicon-like iron ion, Fe XIII, is significantly present at hotter coronal temperatures $\left(T_{\mathrm{e}} \sim 1.6 \mathrm{MK}\right)$, and several strong emission lines appear mostly in the EIS shorter-wavelength observing band, all originating from $3 s^{2} 3 p^{2}-3 s^{2} 3 p 3 d$ transitions. We investigated diagnostic capabilities, especially for density, by a detailed comparison of data from a solar active region, a solar flare, and LHD/EBIT (electron beam ion trap) experiments with values from theoretical calculations $[13,16]$.

The LHD experiment indicates that the value of the line intensity ratio, Fe XIII $\lambda 203.8 / \lambda 202.0$, at the highdensity limit is somewhat below 4 , while the EBIT experiment indicates that it is $\geq 4$, and EIS observations of the 2007 Jan 16 flare shows $\sim 4.3$ (see Fig. 3). This discrepancy between the laboratory measurements and solar observations is attributed to the oxygen lines formed in LHD possibly being blended with Fe XIII $\lambda 202.0$. In solar observations, intensity maps of emission lines formed at various temperatures were compared with that of Fe XIII, and
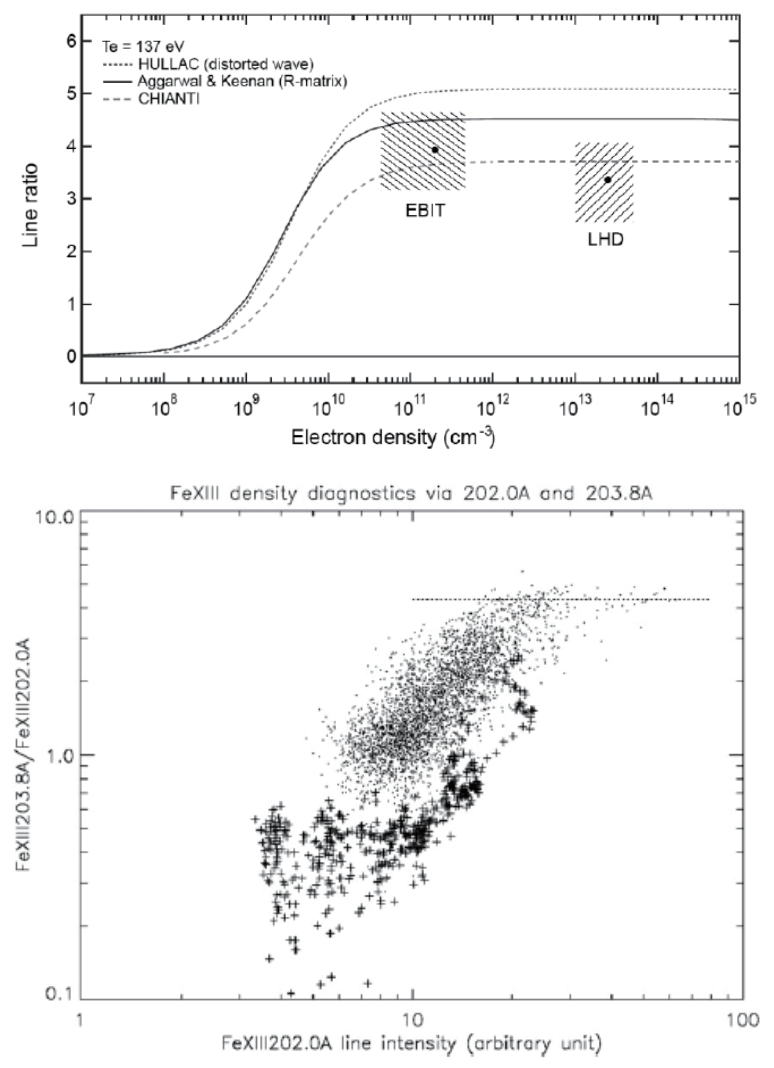

Fig. 3 Fe XIII $\lambda 203.8 \mathrm{~A} / \lambda 202.0$ line ratios: (top panel) laboratory measurements, (bottom) from a small flare $(2007$ January 16 (dots) and AR10921 (2006 November 6) (+) in the same arbitrary intensity units. The dotted horizontal line shows the average value of 4.34 obtained from the data points exceeding 40 in the intensity units $[13,16]$.

the conclusion is that solar $\lambda 203.8$ line is unblended and that the solar measurements are in agreement with the Y08 theoretical model.

Four line pairs $(\lambda 196.5 / \lambda 202.0, \lambda 200.0 / \lambda 202.0$, $\lambda 203.2 / \lambda 202.0, \lambda 203.8 / \lambda 202.0)$ give consistent densities for the EIS AR observation $\left(n_{\mathrm{e}} \sim 10^{8.5-9.5} \mathrm{~cm}^{-3}\right)$. The Y08 model successfully give slightly better consistency between the ratios than the CHIANTI model for these strong line pairs. For other Fe XIII lines, however, problems are found with both of the theoretical models [16].

\subsection{Case study - Fe XVII for intensity branching ratio -}

$\mathrm{Ne}$-sequence iron ions, Fe XVII, are formed around the temperature of $\log T_{\mathrm{e}} \sim 6.7$, which near the maximum temperature reached in solar active regions. The EIS instrument has detected several weak Fe XVII emission lines appearing in both the shorter and longer wavelength bands, and they are identified as those from the transitions between $2 p^{5} 3 \mathrm{~s} / 3 \mathrm{p}-2 \mathrm{p}^{5} 3 \mathrm{p} / 3 \mathrm{~d}$. In particular, the $\lambda 204.6$ and $\lambda 254.8$ lines have a common upper level, $2 \mathrm{~s}^{2} 2 \mathrm{p}^{5} 3 \mathrm{p}{ }^{1} \mathrm{~S}_{0}$, and the intensity ratio solely depends on the branching ratio determined by quantum physical processes.

Warren et al. (2008) [19] found that the observed in- 

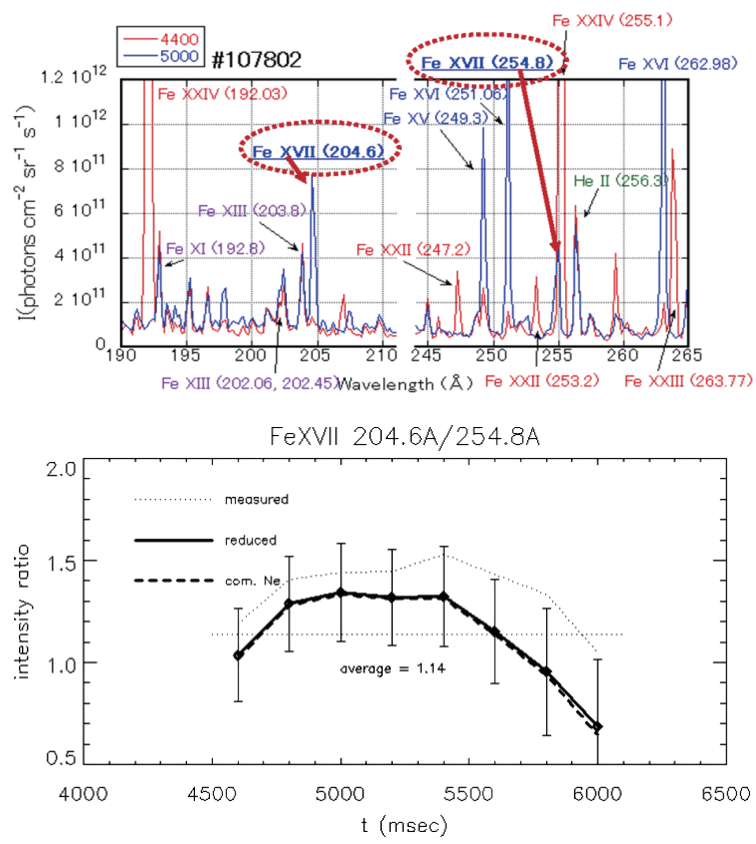

Fig. 4 a) Snapshot of EUV spectra for highly charged iron ions created in LHD: Strong Fe XXIV lines dominate around $T_{\mathrm{e}} \sim 2 \mathrm{keV}(t=4,400 \mathrm{msec})$, while Fe XVII emission appears prominently after the injection of iron TESPEL with the tuning of NBI $(t=5,000 \mathrm{msec}), \mathrm{b})$ Time series of the line intensity ratio $\lambda 204.6 / \lambda 254.8$. The dotted line indicates the measured ratios. The solid line is the reduced Fe XVII line ratio with the intensities of blended Fe XII, XIII lines subtracted from the total line intensities at $\lambda 204.6$. The dashed lines are derived by assuming the same electron density for Fe XII, and Fe XIII lines.

tensity branching ratio of the transitions to the $2 \mathrm{p}^{5} 3 \mathrm{~s}\left({ }^{1,3} \mathrm{P}_{0}\right)$ levels were inconsistent with the theoretical predictions by a factor of $\sim 2$, while old SKYLAB data recorded on Xray film in the S082A experiment at that time indicates that the ratio should be $\sim 1.13$, similar to theoretical predictions [20]. Del Zanna and Ishikawa (2009) [21] noticed that the Fe XVII $\lambda 204.6$ line was blended by emission lines originating from the TRs. The intensity ratio of these lines having a common upper level was successfully measured by LHD, and we obtained the experimental value of $\sim 1.1$, clearly $\leq 1.3$ by eliminating the contribution of blended $\mathrm{Fe}$ XII and Fe XIII lines emerging near the 1204.6 line (see Fig. 4).

Re-analysis of data from the core of an AR and from a small flaring event on 2007 June 2 reveals that the intensity ratio significantly reduces to $\sim 1.5-1.6$, by considering TR line blending for the $\lambda 204.6$ line. This is still systematically higher than the experimental value. Theoretical estimation of the branching ratio has also been revisited recently, but the error range of theoretical predictions has not been found compatible with the discrepancy [22]. The experimental and theoretical confirmation of the Fe XVII intensity branching ratio has consequence for the in-flight calibration of the EIS instrument; the calibration should be revisited more in detail.

\subsection{Case study of Fe XXII, and prospects for density diagnostics for solar high- temperature plasmas}

The excitation processes of highly charged iron ions, namely Fe XIX - Fe XXII ions, were also investigated at the LHD and EBIT facilities [23]. Fe XXII densitysensitive line ratios were analyzed with several CR models, including ours, and these experimental data can be reproduced in the analysis when thermal proton impact excitation rates are taken into account. Those ions mainly produce emission lines outside the EIS observing wavelengths, so direct comparison with solar spectra is not feasible at the moment. However, the EUV spectroscopic telescope (EUVST) is expected to be on board the Solar-C satellite, which is a next-generation Sun observing spacecraft [24]. The increased spectroscopic capability of EUVST will enable high-resolution, both in spatial and spectral domains, and high-cadence observations of EUV/UV emission lines originating from Fe XVIII - Fe XXIV ions. In addition density information of $T_{\mathrm{e}} \sim 10 \mathrm{MK}$ plasma, obtained available from Fe XXI lines in the EUV channel will be very useful.

\section{Coupling with Solar Atmospheric Dynamics}

We have improved atomic parameters compiled in our CR model by analyzing and evaluating experimental and observational data compared with theoretical predictions. Direct application to non-equilibrium plasmas in the solar atmosphere requires combining the $\mathrm{CR}$ model with the models for the dynamics of the solar atmosphere. Two simple steady-state dynamics models have been developed so far $[25,26]$.

The effect of time-dependent ionization and recombination processes on magnetic reconnection in the solar corona has been investigated, especially for Petschek [9]type steady reconnection, in which the magnetic energy is converted to heat mainly by slow-mode shocks. We have calculated the transient ionization of iron, with the other species being assumed in ionization equilibrium. Specific iron line emissions have also been calculated for comparison with observations [25].

The other case is applied for a transient coronal hole associated with an X-class flare of 2006 Dec 13. Temperature-dependent upflows are observed in the dimming region following a coronal mass ejection, and the data obtained from this region gives some boundary conditions in the dynamics modeling. It is found that the intensity in the dimming region dramatically drops within 30 minutes from the flare onset, and the dimming region reaches the equilibrium stage after $\sim 1 \mathrm{hr}$. The temperature-dependent upflows are observed during this equilibrium stage by the EIS instrument. The dynamics model is constructed from the mass and momentum conservation equations to demonstrate the height variation of 
plasma conditions in the dimming region. A super-radial expansion of the magnetic field of the flow is required for the mass and momentum conservation, and a steep temperature and velocity gradient at around $7 \mathrm{Mm}$ from the solar surface is needed in the dynamics model. This result may suggest that the strong heating occurred at this height. The assumption of ionization equilibrium is violated in the dimming region, particularly at higher temperature layers. A rapid drop of electron density with height triggers significant deviation from the ionization equilibrium state. Plasma density information is crucial to understand the behavior of this transient phenomenon, which unfortunately could not be obtained directly from density sensitive line ratios in the study [26].

\section{Summary}

EUV emission lines originating from highly charged iron ions provide much information about hot plasmas in the universe, and a time-dependent CR model with the best available set of physical parameters for iron ions is a useful tool to analyze these plasmas. Cross validation of nonequilibrium modeling can be performed with solar spectroscopic observations and LHD laboratory measurements, combining appropriate models of dynamics.

\section{Acknowledgement}

Hinode is a Japanese mission developed and launched by ISAS/JAXA, collaborating with NAOJ as domestic partner, NASA and STFC (UK) as international partners. Scientific operation of the Hinode mission is conducted by the Hinode science team organized at ISAS/JAXA. This team mainly consists of scientists from institutes in the partner countries. Support for the post-launch operation is provided by JAXA and NAOJ (Japan), STFC (U.K.), NASA (U.S.A.), ESA, and NSC (Norway). This work is partly carried out at the NAOJ Hinode Science Center, which was supported by the Grant-in-Aid for Creative Scientific Research: "The Basic Study of Space Weather Prediction" from MEXT, Japan (Head Investigator: K. Shibata), generous donations from Sun Microsystems, and NAOJ/NINS internal funding. It was also supported by NINS inter-institute collaborative program for Creation of New Research Area (Head Investigator: T. Watanabe), by NIFS/NINS under the project of Formation of International Network for Scientific Collaborations (Head Investigator: H. Yamada), and by NIFS collaboration research programs (NIFS10KLPF008 and NIFS12KLPF027). We also thank Dr. A. C. Sterling for improving of the manuscript for better expressions.

[1] T. Watanabe, T. Kato, I. Murakami and N. Yamamoto, in AIP Conf. Proc. 901, 5th International Conference on Atomic and Molecular Data and Their Applications
(ICAMDATA) (Melville: AIP) p.215 (2007).

[2] T. Watanabe and the EIS Team, Plasma Fusion Res. 2, S10011 (2007).

[3] C.M. Brown, U. Feldman, J.F. Seely and C.M. Korendyke, Astrophys. J. Suppl. 176, 511 (2008).

[4] E. Landi, G. Del Zanna, P.R. Young, K.P. Dere, H.E. Mason and M. Landini, Astrophys. J. Suppl. 162, 261 (2006).

[5] H. Hara, T. Watanabe, L.K. Harra, J.L. Culhane, P.R. Young, J.T. Mariska and G.A. Doschek, Astrophys. J. Lett. 678, L67 (2008).

[6] G.A. Doschek, H.P. Warren, J.T. Mariska, K. Muglach, J.L. Culhane, H. Hara and T. Watanabe, Astrophys. J. 686, 1362 (2008).

[7] B. De Pontieu, S.W. McIntosh, V.H. Hansteen and C.J. Schrijver, Astrophys. J. Lett. 701, L1 (2009).

[8] H. Hara, T. Watanabe, L.K. Harra, J.L. Culhane and P.R. Young, Astrophys. J. 741, 107 (2011).

[9] H.E. Petschek, in "The Physics of Solar Flares," ed. W. N. Hess, NASA SP-50 (NASA, Washington, D.C.), 425 (1964).

[10] M.B. Chowdhuria, S. Morita, M. Goto, H. Nishimura, K. Nagai and S. Fujioka, Rev. Sci. Instrum. 78, 023501 (2007).

[11] A. Bar-Shalom, M. Klapisch and J. Oreg, J. Quant. Spectrose. Radiat. Transf. 71, 179 (2001).

[12] K.M. Aggarwal and F.P. Keenan, Astron. Astrophys. 418, 371 (2004).

[13] N. Yamamoto, T. Kato, H. Funaba, K Sato, N. Tamura, S. Sudo, P. Beiersdorfer and J.K. Lepson, Astrophys. J. 689, 646 (2008) [Y08].

[14] K.M. Aggarwal and F.P. Keenan, Astron. Astrophys. 429, 1117 (2005).

[15] F.P. Keenan, D.B. Jess, K.M. Aggarwal, J.W. Brosius and J.M. Davila, Mon. Not. R. Astron. Soc. 376, 205 (2007).

[16] T. Watanabe, H. Hara, N. Yamamoto, D. Kato, H.A. Sakaue, I. Murakami, T. Kato, N. Nakamura and P.R. Young, Astrophys. J. 692, 1294 (2009).

[17] I. Skobelev, I. Murakami and T. Kato, National Institute for Fusion Science Research Report, NIFS-DATA-95, 1 (2006).

[18] I. Skobelev, I. Murakami and T. Kato, National Institute for Fusion Science Research Report, NIFS-DATA-99, 1 (2007).

[19] H.P. Warren, U. Feldman and C.M. Brown, Astrophys. J. 685, 1277 (2008).

[20] G.A. Doschek, U. Feldman and A.K. Bhatia, Phys. Rev. A 43(5), 2565 (1991)

[21] G. Del Zanna and Y. Ishikawa, Astron. Astrophys. 508, 1517 (2009).

[22] P. Jönsson et al., National Institute for Fusion Science Research Report, NIFS-DATA-113, 1 (2011).

[23] H.A. Sakaue, N. Yamamoto, S. Morita, N. Nakamura, C. Chen, D. Kato, H. Kikuchi I. Murakami, S. Ohtani, H. Tanuma, T. Watanabe and H. Tawara, J. Appl. Phys. 109, 073304 (2011).

[24] Solar-C Working Group, "Interim Report on the Solar-C Mission Concept, Plan-B," Chap. 3 (2011).

[25] S. Imada, I. Murakami, T. Watanabe, H. Hara and T. Shimizu, Astrophys. J. 742, 70 (2011).

[26] S. Imada, H. Hara, T. Watanabe, I. Murakami, L.K. Harra, T. Shimizu and E.G. Zweibel, Astrophys. J. 743, 57 (2011). 